\title{
Localization Using a Mobile Beacon with Directional Antenna for Wireless Sensor Networks
}

\author{
Yao-Hung $\mathrm{WU}^{\dagger}$, Nonmember and Wei-Mei $\mathrm{CHEN}^{\dagger \text { a) }}$,Member
}

\begin{abstract}
SUMMARY Wireless sensor networks are comprised of several sensor nodes that communicate via wireless technology. Locating the sensor nodes is a fundamental problem in developing applications for wireless sensor networks. In this paper, we introduce a distributed localization scheme, called the Rectangle Overlapping Approach (ROA), using a mobile beacon with GPS and a directional antenna. The node locations are computed by performing simple operations that rely on the rotation angle and position of the mobile beacon. Simulation results show that the proposed scheme is very efficient and that the node positions can be determined accurately when the beacon follows a random waypoint movement model.

key words: wireless sensor networking, localization, mobile beacon, directional antenna, GPS
\end{abstract}

\section{Introduction}

Wireless sensor networks (WSNs) have wide application in fields such as environmental observation, military monitoring, and disaster relief [2]. Recent advances in wireless communications and electronics have enabled the development of small low-cost sensor nodes that communicate over short distances. Wireless sensor networks are comprised of several sensor nodes that communicate via wireless technology. Each node can sense certain aspects of its environment and perform limited computations. Node volume, electrical capacity and data storage capability restrict node operation in WSNs. Thus, many researchers concentrated on the development of scalable, low-cost, and low-power consumption WSNs.

Localization is one of the most important topics in WSN research. An accurate localization scheme can support transmission of the sensor position information, routing [3] and technology services. So far, many localization methods have been presented that could be implemented using one or a few powerful nodes with Global Positioning System (GPS). GPS coordinates are a good estimate of sensor position, but it is too expensive to equip each sensor with GPS. A feasible solution is to designate a small number of sensor nodes as anchor points or beacons and equip them with GPS. They can help other sensor nodes locate themselves using the information broadcast by the beacons.

In this paper, we present a simple and efficient localization scheme based on a self-propelled device equipped with

\footnotetext{
Manuscript received December 27, 2010.

Manuscript revised June 10, 2011.

${ }^{\dagger}$ The authors are with the Department of Electronic Engineering of National Taiwan University of Science and Technology, Taiwan.

a)E-mail: wmchen@mail.ntust.edu.tw (corresponding author) DOI: 10.1587/transinf.E94.D.2370
}

GPS and a directional antenna. Distributed computation of the positions is performed by simple operations on information received from the moving beacon. In Sect. 2, we review the related work on localization in WSNs. The proposed localization scheme and the simulation results are presented in Sects. 3 and 4. Conclusions are discussed in Sect. 5.

\section{Related Work}

Recently, many localization schemes for WSNs have been presented. These localization algorithms [5], [13] fall into two categories: range-based and range-free schemes.

Range-based schemes are usually supported by additional sensor hardware that measures the distances or angles of the signals being transmitted between the nodes. Range-based schemes determine the distance between two sensor nodes based on information such as Time of Arrival (TOA) [16], Time Difference of Arrival (TDOA) [17], Angle of Arrival (AOA) [14], and Received Signal Strength Indicator (RSSI) [4]. After the relative distance is determined, it is simple to compute the location of each node. The drawbacks of range-based schemes are easier to be interfered by multipath, fading, and noise and an additional hardware is required.

On the other hand, range-free schemes avoid costly hardware by exploiting inter-node communication and the sensing range of the node to estimate the positions of the sensor nodes. Lately many range-free localization schemes have been proposed, such as Centroid [7], DV-HOP [15], and APIT [11]. Centroid scheme calculates the locations of sensor nodes based on reference information that is broadcast by specific stationary points. DV-HOP scheme measures hop counts from each node to specific anchor points to localize the nodes' positions. In APIT scheme, each node estimates whether it resides inside or outside several triangular regions bounded by the locations that are received from the anchor points, and refines the location by overlapping the regions of the sensor resided. Some area-based localization mechanisms are developed to narrow down the possible region for a sensor node [8], [10]. Sextant [10] operates by setting up and solving a system of geographic constraints based on connectivity information from the underlying communication network and achieves high accuracy by enabling non-convex constraints to refine position estimates. The above approaches typically need a large amount of stationary anchor points involved a heavy computation for achieving higher accuracy. Besides, extensive communi- 
cation among neighboring sensor nodes brings a high traffic load.

Mobile localization exploits moving beacons to avoid the problems of static range-free localization schemes. A sensor node narrows down the possible region to estimate the location based on the mobile beacon message [9], [19]. Xiao et al. proposes another scheme using the arrival and departure overlap area, which is a possible area delimited by two circles with the same radius at different centers [20]. Ssu et al. introduces scheme [18] that the location of a sensor node is estimated using the intersection points of two perpendicular bisectors of the chords obtained by three beacon points. To improve the localization accuracy of Ssu's scheme, an efficient scheme is suggested to estimate sensor locations from possible areas by using geometric constraints [12].

In the next section, we introduce a localization scheme, called Rectangle Overlapping Approach (ROA), to locate sensor nodes efficiently using a mobile beacon with GPS and a directional antenna. After the mobile beacon traverses a predefined route, each sensor node can estimate its location by simple distributed computations. Each sensor node receives the messages but does not interact with other nodes for localization. Distributed computation of node position involving elementary operations allows the system to operate in a low power consumption mode.

\section{Rectangle Overlapping Approach (ROA)}

The ROA scheme is based on a self-propelled device that can move along a predefined route and is equipped with GPS and a directional antenna. After traversing a predefined route, the mobile beacon can help complete the localization of all sensor nodes.

\subsection{The Model of the ROA Scheme}

We assume that the transmission range of each sensor node is a circle with radius $r$. We do not discuss collision problems because we assume that they can be solved by the MAC layer protocols. In addition, we assume that the sensor nodes are deployed in a square of side Range as shown in Fig. 1. We set the coordinates of the upper left corner at $(0,0)$ and those of the lower right corner at (Range, Range).

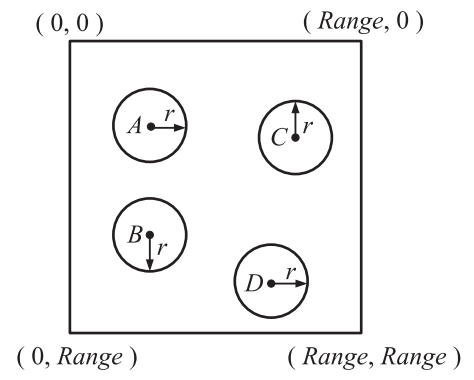

Fig. 1 The coordinator plane of Range $\times$ Range involves sensor nodes $A$, $B, C$, and $D$ with transmission radius $r$.
Antenna models are classified into two types according to beam pattern: omni-directional antenna and directional antenna. The radiated signal from an omni-directional antenna is easier to be interfered by environment noise. A directional antenna concentrates energy on a particular narrow direction with a higher gain, reducing interference to environment noise can improve localization accuracy. By using directional antenna, a sensor node may selectively receive signals only from a certain desired direction. This feature supports our scheme to narrow down the possible region more quickly and to improve the accuracy of localization. As in the network model [1], we assume that the mobile beacon is capable of transmitting directionally. The directional antenna at the mobile beacon should have a narrow beamwidth that can be rotated in any direction. For the purpose of this paper, we simplify the antenna transmission pattern by representing it as a narrow sector of angle $\Delta \theta$ as shown in Fig. 2.

We assume that the sensing range of the mobile beacon is also a circle with radius $r$. The beacon broadcasts messages periodically as it traverses its predefined route at a constant velocity $v$. The mobile beacon transmission interval is the time between two consecutive beacon messages. It is denoted as $\Delta t$. The beacon message contains the location of the beacon. The directional antenna of the beacon rotates a full circle counterclockwise by periodically turning $n$ times. Thus, each rotation angle is $n=360^{\circ} / \Delta \theta$ where $\Delta \theta$ divides 90 . When the system begins to operate, the mobile beacon first broadcasts two important parameters, $r$ and $\Delta \theta$ to all sensor nodes. Each node stores these two parameters in its local memory for later computation.

Each phase involves the beacon moving a fixed distance $v \Delta \mathrm{t}$. After stopping, the beacon periodically rotates an angle $\Delta \theta n$ times while transmitting signals through its directional antenna. Since the beacon is position-aware, it will broadcasts two important parameters: $i$ and $\left(x_{b}, y_{b}\right)$, where $i$ means the $i$ th rotation in a round for $1 \leq i \leq n$ and $\left(x_{b}, y_{b}\right)$ is the current position of the beacon.

The position of the beacon defines the center of a circle of radius $r$. When a sensor node receives the value of $i$, it knows that its position is located in the sector between $(i-1) \Delta \theta$ and $i \Delta \theta$. For example, as shown in Fig. 3, if $\Delta \theta=$ $30^{\circ}$ and the sensor node receives $i=2$, then the sensor node knows its position is located in a sector between $30^{\circ}$ and

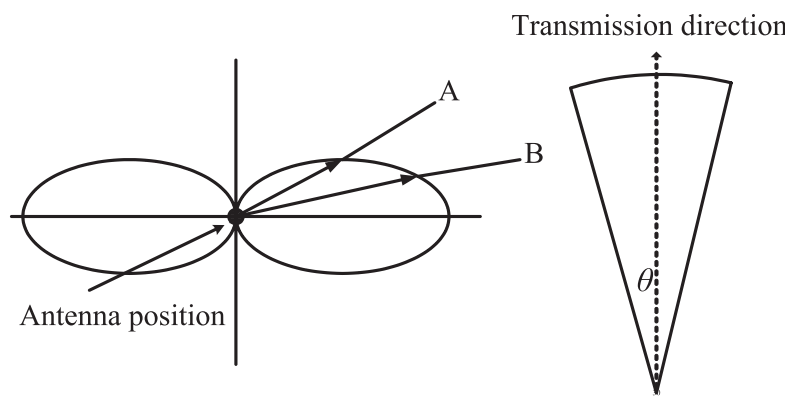

Fig. 2 Antenna model 


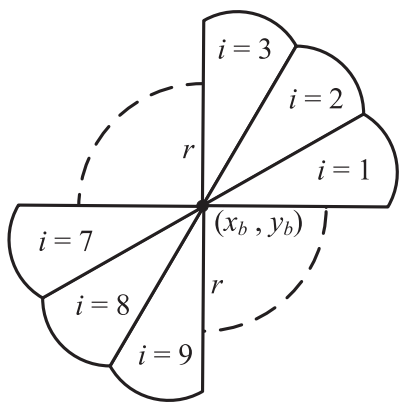

Fig. 3 The sectors through directional antenna with $\Delta \theta=30^{\circ}$.

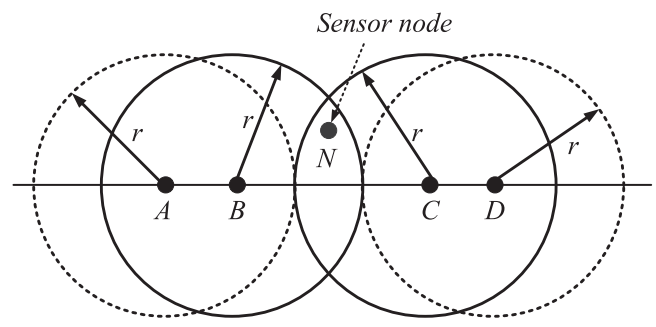

Fig. 4 The state information of the mobile beacon.

$60^{\circ}$. That is,

$(i-1) \Delta \theta \leq$ the angle of the $i$ th rotation $<i \Delta \theta$.

After the mobile beacon rotates one full circle counterclockwise, the beacon moves to the next position along the predefined route.

\subsection{Arrival and Departure of a Mobile Beacon}

The four states of a sensor node we need to define are:

- In: The node is within the transmission range of the mobile beacon.

- Out: The node is outside the transmission range of the mobile beacon.

- Arrival: The node has entered the state in from the state out.

- Departure: The sensor node has entered the state out from the state in.

As shown in Fig. 4, the sensor node $N$ is in the state in when the beacon is at point $B$ or $C$ and is in the state out when the beacon is at point $A$ or $D$. Furthermore, the sensor node $N$ is in the state arrival when the beacon moves from point $A$ to $B$ and is in the state departure when the beacon moves from point $C$ to $D$.

\subsection{The Description of the ROA Scheme}

When a sensor node receives the signal from the directional antenna of the mobile beacon for the first time, the sensor node generates a sector of radius $r$. This sector and the circle will have two intersection points, denoted by $P_{1}$ and $P_{2}$ in Fig. 5. The center of the mobile beacon can be considered the new origin $\left(x_{b}, y_{b}\right)$. Point $P_{1}$ is defined as the first

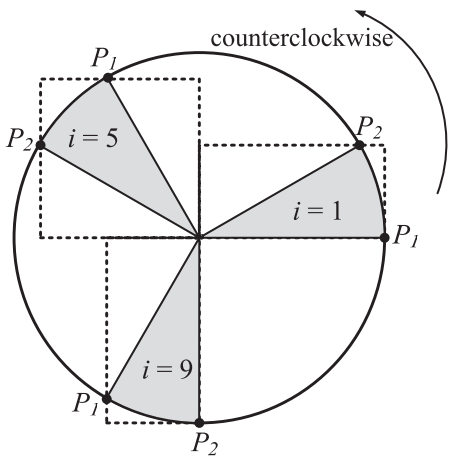

Fig. 5 The ER zones, $P_{1}$ and $P_{2}$ points with $\Delta \theta=30 \circ$ and $i=1,5$, and 9.

point crossed in a counterclockwise rotation about this origin. Point $P_{2}$ is the second intersection point crossed during this rotation. Figure 5 shows the positions of $P_{1}$ and $P_{2}$ viewed in each quadrant of the coordinate plane. If a sensor node receives $i$ and $\left(x_{b}, y_{b}\right)$ from the beacon, the coordinates of points $P_{1}\left(x_{1}, y_{1}\right)$ and $P_{2}\left(x_{2}, y_{2}\right)$ can be computed:

$$
\left(x_{1}, y_{1}\right)=\left(x_{b}+r \cos ((i-1) \Delta \theta), y_{b}-r \sin ((i-1) \Delta \theta)\right)
$$

and

$$
\left(x_{2}, y_{2}\right)=\left(x_{b}+r \cos (i \Delta \theta), y_{b}-r \sin (i \Delta \theta)\right) \text {. }
$$

A vertical line through $P_{1}$ and a horizontal line through $P_{2}$ will form a rectangle with the $x$ - and $y$-axes that contains the position of the mobile beacon as shown in Fig. 5. We call this rectangle the ER (Estimated Rectangle).

$$
\mathrm{ER}=\left\{(x, y) \mid x_{\min } \leq x \leq x_{\max }, y_{\min } \leq y \leq y_{\max }\right\}
$$

Each ER is identified by four endpoints, $x_{\min }, x_{\max }, y_{\min }$ and $y_{\max }$ that are generated as follows:

- If $0^{\circ} \leq i \Delta \theta<90^{\circ}$,

$$
x_{\min }=x_{b}, x_{\max }=x_{1}, y_{\min }=y_{2}, \text { and } y_{\max }=y_{b}
$$

- If $90^{\circ} \leq i \Delta \theta<180^{\circ}$,

$$
x_{\min }=x_{2}, x_{\max }=x_{b}, y_{\min }=y_{1}, \text { and } y_{\max }=y_{b}
$$

- If $180^{\circ} \leq i \Delta \theta<270^{\circ}$,

$$
x_{\min }=x_{1}, x_{\max }=x_{b}, y_{\min }=y_{b}, \text { and } y_{\max }=y_{2}
$$

- If $270^{\circ} \leq i \Delta \theta<360^{\circ}$,

$$
x_{\min }=x_{b}, x_{\max }=x_{2}, y_{\min }=y_{b}, \text { and } y_{\max }=y_{1} .
$$

Each sensor node records two sets of ER values which are called ER and ER' respectively. We set the initial ER value as Range $\times$ Range, so the first ER is also the largest,

$$
\mathrm{ER}=\{(x, y) \mid 0 \leq x \leq \text { Range, } 0 \leq y \leq \text { Range }\} .
$$

Besides recording this first ER, the sensor node also needs to record the value of ER'. In the arrival state, each sensor node generates a new ER' from the beacon interval. 

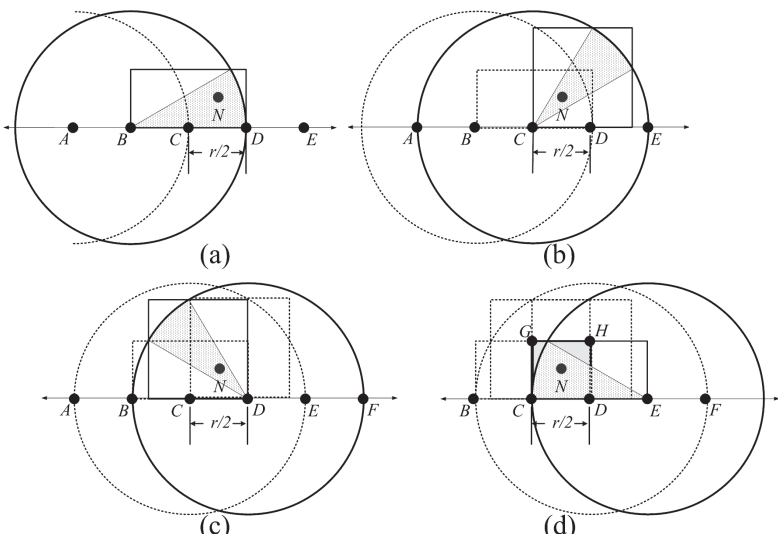

(d)

Fig. 6 The operating process of ROA scheme.

If the sensor node is still in the in state, it will replace the previous ER'. After entering the departure state, we find the intersection of ER and ER' and generate a new ER to replace the old ER. Repeating this process does not converge before a suitably small rectangle is generated.

The steps of the ROA scheme are illustrated in Fig. 6. First, the beacon is at position $A$ and the sensor node is in the out state. When the beacon arrives at position $B$, we set the state of the sensor node to arrival and generate ER'. When the beacon arrives at the next position $C$, we create a new ER' and replace the previous ER'. Finally, when the beacon arrives at position $F$, the state of the sensor node is set to departure. We then find the intersection of ER and ER', generate a new ER and designate the centroid of this new ER to be the estimated localization position.

The ROA scheme is described in the following.

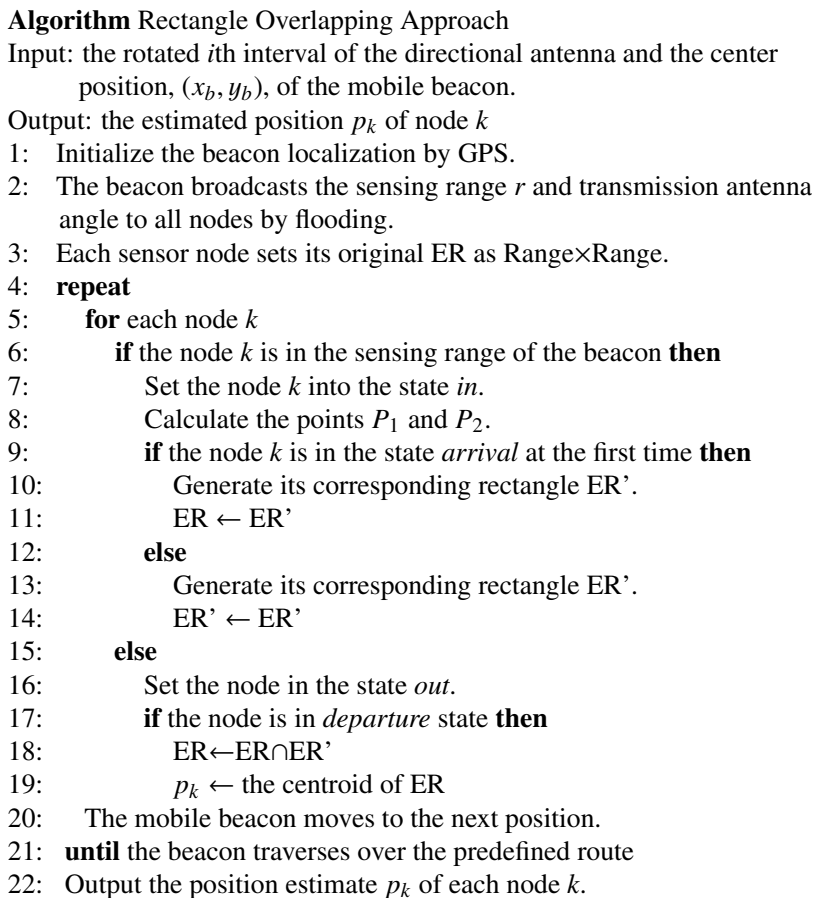

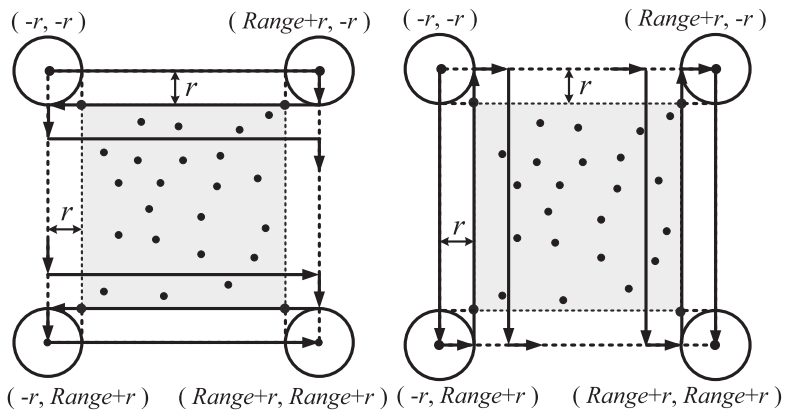

(a) a horizontal pattern

(b) a vertical pattern

Fig. 7 The line scanning movement.

\subsection{Movement Pattern of a Mobile Beacon}

In this section, we discuss the traversing strategy for a beacon. There are two movement patterns we consider:

- Line scanning movement: This movement pattern involves the beacon completing a series of horizontal movements followed by a series of vertical movements. In the ROA scheme, a sensor node changing from the out to in state, can theoretically estimate position more accurately. We design the beacon to move from $(-r,-r)$ to $($ Range $+r$, Range $+r$ ) along horizontal straight lines following a route that passes through certain significant points $(-r,-r) \rightarrow \cdots \rightarrow($ Range $+r,-r)$ $\rightarrow \cdots \rightarrow(-r$, Range $+r) \rightarrow \cdots \rightarrow($ Range $+r$, Range $+r)$ and to reverse vertically as shown in Fig. 7. The total number of movements is

$$
2\left\lfloor\frac{\text { Range }+2 r}{v \Delta t}\right\rfloor^{2} \text {. }
$$

The results of the simulation indicate that a single horizontal traverse may allow the beacon to obtain a high localization accuracy. This could save the beacon half of its operating power consumption.

- Random waypoint movement [6]: The mobile beacon randomly selects a destination in the sensor field and moves to that location at a constant velocity. When the mobile beacon arrives at this destination, the mobile beacon continues travelling to the next destination at the same constant speed.

\subsection{The Analysis of Error}

If the actual position of the sensor node is $(x, y)$, the estimated position of the node is $\left(x^{\prime}, y^{\prime}\right)$. We estimate this position as

$$
\left(x^{\prime}, y^{\prime}\right)=\left(\frac{x_{\min }+x_{\max }}{2}, \frac{y_{\min }+y_{\max }}{2}\right),
$$

where $x_{\min }, x_{\max }, y_{\min }$, and $y_{\max }$ are ER boundary points. As in Fig. 6, we can show that the maximum horizontal error occurs at the intersection of the final ER of side length $r$ if 
the moving distance is in the range $1 \leq v \Delta t \leq r$. If $\left(x_{b}, y_{b}\right)$ is the center of the mobile beacon, we can derive the horizontal and vertical errors of the ROA scheme as

$$
\begin{aligned}
& \text { Error }_{x}=\left|x-x^{\prime}\right| \leq \frac{x_{b}+r-x_{b}}{2} \leq \frac{r}{2} \text { and } \\
& \text { Error }_{y}=\left|y-y^{\prime}\right| \leq \frac{y_{b}+r-y_{b}}{2} \leq \frac{r}{2} .
\end{aligned}
$$

Thus, we have that the maximum error of the estimated position of a sensor node is $\sqrt{2} r / 2 \cong 0.7 r$.

However, the directional antenna is a separate source of error. Antenna angel error is defined as an error in the beam width of the directional antenna. We define the error zone and undefined zone as areas in which sensor nodes may be susceptible to localization error, as shown in Fig. 8. The beacon $M$ traverses the line and three sensor nodes $A, B$, and $C$ receive messages from beacon $M$ by directional antenna with $\Delta \theta=30^{\circ}$. In this area, sensor nodes $B$ and $C$ will receive the rotation parameter $i=2$ and node $A$ will receive $i=3$. Unfortunately, if the directional antenna has a beam angle error $\Delta \theta$, node $A$ will receive $i=2$ and nodes $B$ and $C$ will receive $i=1$. After the beacon moves a further distance $r$, the sensor at node $A$ will no longer have an intersection with the beacon. However, nodes $B$ and $C$ may proceed normally. Thus, sensor node $A$ created an ER which did not include itself in this range. We posited that the correct position of node $A$ is $P(x, y)$ and the new error position is $P^{\prime}\left(x^{\prime}, y^{\prime}\right)$ with the following coordinates:

$$
\begin{aligned}
& x=\frac{x_{b}+r \cos ((i-1) \Delta \theta}{2}, \\
& y=\frac{y_{b}-r \sin ((i-1) \Delta \theta}{2}, \\
& x^{\prime}=\frac{x_{b}+r \cos ((i-2) \Delta \theta}{2}, \text { and } \\
& y^{\prime}=\frac{y_{b}-r \sin ((i-2) \Delta \theta}{2} .
\end{aligned}
$$

So, we derive the error distances as:

$$
\text { Error }_{x}=\frac{r}{2}|\cos ((i-1) \Delta \theta)-\cos ((i-2) \Delta \theta)| \text { and }
$$

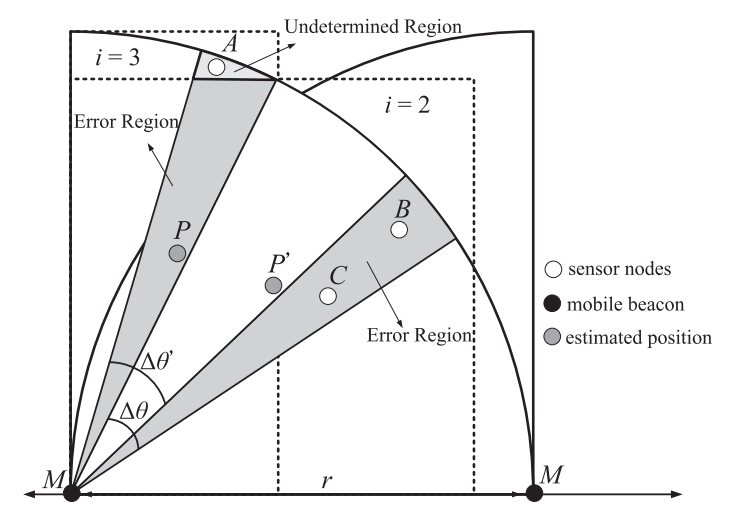

Fig. 8 Radio propagation pattern.

$$
\text { Error }_{y}=\frac{r}{2}|\sin ((i-1) \Delta \theta)-\sin ((i-2) \Delta \theta)| \text {. }
$$

By numerical computation, Error $x \leq r / 2$ and Error $_{y} \leq r / 2$.

According to this analysis, the two values radius $r$ and directional antenna error $\Delta \theta$ are important factors in localization error. Decreasing both values may improve the localization accuracy by reducing certain localization error. This analysis was also verified by the results of the simulation.

\section{Performance Evaluation}

In this section, we verify the accuracy and reliability of the ROA scheme by simulation and compare the performance with several well-known range-free localization schemes.

\subsection{Simulation Environment and Parameters}

Our simulation was built on the MATLAB simulator. We do not discuss collisions because we assume they can be solved by the MAC layer protocols and the signals can only be received in the LOS propagation. Each sensor node is forced to stay active during the localization process. The sensor nodes are deployed in an effective space range of $300 \mathrm{~m} \times 300 \mathrm{~m}$ without obstacles and 100 sensor nodes are randomly deployed. The radio propagation model assumed free space. The parameter settings for the ROA simulations are shown in Table 1. We performed this simulation with various parameters to get a comprehensible result.

\subsection{Metrics}

Three metrics are employed to evaluate the performance of our localization algorithm.

- Average localization error: It is defined as the average difference between estimated location $\left(x^{\prime}, y^{\prime}\right)$ and actual location $(x, y)$ of all sensor nodes

$$
\Delta E_{a v}=\frac{1}{N} \sum_{i=1}^{N} \sqrt{\left(x-x^{\prime}\right)^{2}+\left(y-y^{\prime}\right)^{2}},
$$

where $N$ denotes the total number of sensor nodes.

- Average execution time: It is defined as the time needed to localize all sensor nodes.

$$
\Delta \mathrm{AT}_{a v}=\frac{1}{N} \sum_{i=1}^{N} t_{i}
$$

where $t_{i}$ denotes the time required for sensor node $i$ to obtain its location.

Table 1 Simulation parameters.

\begin{tabular}{ll}
\hline Parameter & Value(s) \\
\hline Beacon message interval & $0.2,0.4,0.6,0.8,1.0(\mathrm{sec})$ \\
Beacon moving speed & $10,20,30(\mathrm{~m} / \mathrm{sec})$ \\
Beacon traversing trajectory & Line scanning, RWP \\
Beacon DOI & $0,0.2,0.4,0.6,0.8$ \\
Beacon transmission radius & $10,20,30(\mathrm{~m})$ \\
Beacon antenna angle & $10^{\circ}, 15^{\circ}, 30^{\circ}, 45^{\circ}$ \\
\hline
\end{tabular}


- Average throughput: It is defined

$$
\Delta R_{a v}=\frac{1}{N} \sum_{i=1}^{N} R_{i},
$$

where $R_{i}$ denotes the number of packets received by sensor node $i$ to estimate its location.

\subsection{Simulation Results}

We explore six sets of simulations which evaluate the different factors below:

- Radius range $r$ : For beacon speed $v(10 \mathrm{~m} / \mathrm{sec}$, $20 \mathrm{~m} / \mathrm{sec}, 30 \mathrm{~m} / \mathrm{sec}$ ), average localization error versus beacon interval $\Delta t$ for different beacon radius $r(10 \mathrm{~m}$, $20 \mathrm{~m}, 30 \mathrm{~m}$ ) with $\Delta \theta=30^{\circ}$ is shown in Fig. 9. The results show that a small radius $r$ can reduce the localization error for both of traverse trajectories. The reason is that the ER area formed by small beacon radius $r$ intersects a smaller region and therefore gives a more accurate estimated location.

- Directional antenna angle $\Delta \theta$ : Average localization error versus beacon interval for different angles $\Delta \theta\left(10^{\circ}\right.$, $15^{\circ}, 30^{\circ}, 45^{\circ}$ ) with $r=10 \mathrm{~m}$ and $v=10 \mathrm{~m} / \mathrm{sec}$ is shown in Fig. 10. The results show that reducing $\Delta \theta$ can reduce the localization error as much as possible. The reason for this is identical to that for the beacon sensing radius $r$. When $\Delta \theta$ is greater than $45^{\circ}$, the large ER area results in a high localization error. Both trajectories produce the same results.
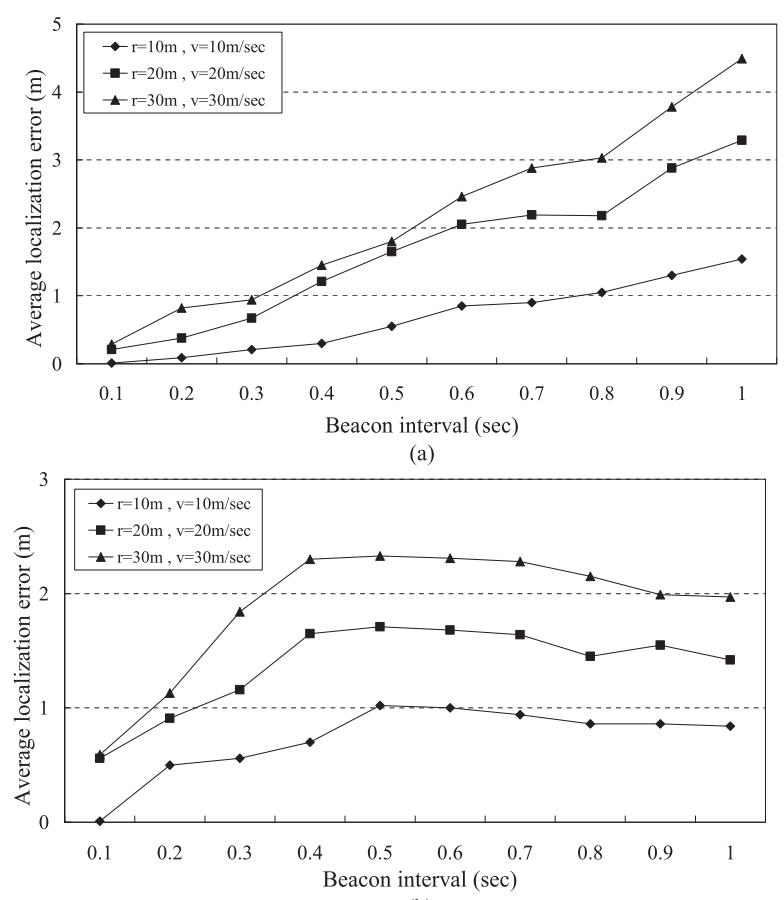

(b)

Fig. 9 Average localization error versus beacon interval for radius $r$ (a) Line scanning (b) Random waypoint.
- Beacon interval $\Delta t$ : Average localization error versus beacon interval is shown in Fig. 9 and Fig. 10. Reducing the interval produces a more accurate estimated position. However, this will require a longer execution time and the transmission of more packets. In our simulation, we observe that moving a distance $v \Delta t$ less than $r / 2$, results in a more accurate estimated location.

- Beacon speed $v$ : A sensor node needs to collect enough beacon messages to localize itself. A beacon with a higher speed must lower its beacon interval. For example, $v=10 \mathrm{~m} / \mathrm{sec}, \Delta t=0.1 \mathrm{sec}$ and $v=20 \mathrm{~m} / \mathrm{sec}, \Delta t=$ $0.05 \mathrm{sec}$. Not all localization schemes are affected by beacon speed.

- Beacon traversing trajectory: In the line scanning model, all sensor nodes are visited but this is time consuming. The random waypoint model completes the sensor node localization more quickly. When $v \Delta t$ is greater than the half of the beacon radius $r$, we adopt a method that doubles the random waypoint's execution time and obtains a better estimated position.

- Degree of irregularity (DOI): To determine whether this scheme is useful in practice, we apply the radio model proposed. In this model, the communication radius of the sensor node is irregular and the notation DOI is used to represent the degree of irregularity. There are the upper bound and the lower bound of the communication radius. The upper bound is the ideal communication radius $r$, and the lower bound is proportional to (1-DOI). Average localization error versus

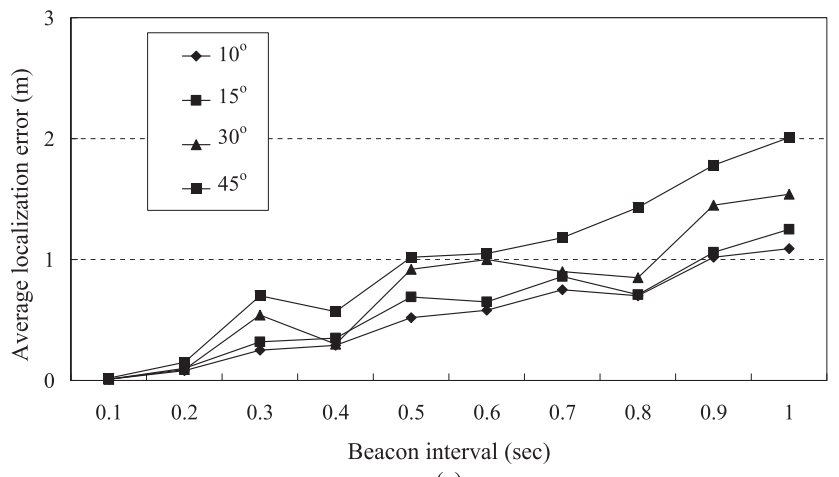

(a)

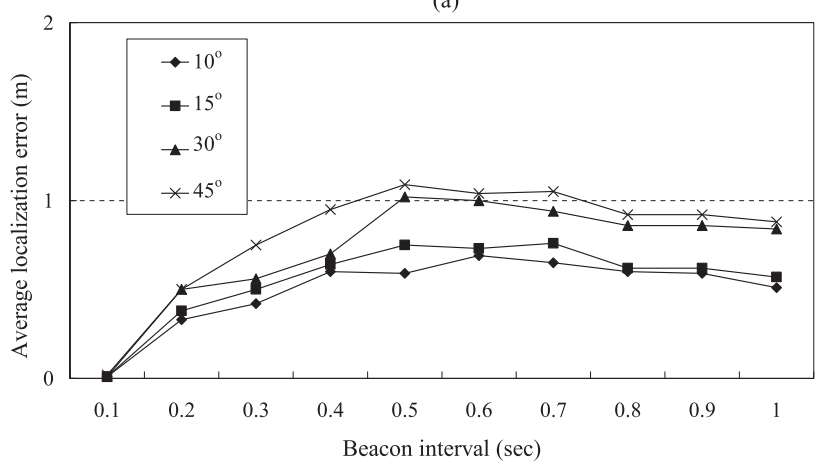

(b)

Fig. 10 Average localization error versus beacon interval for $\Delta \theta$ (a) Line scanning (b) Random waypoint. 


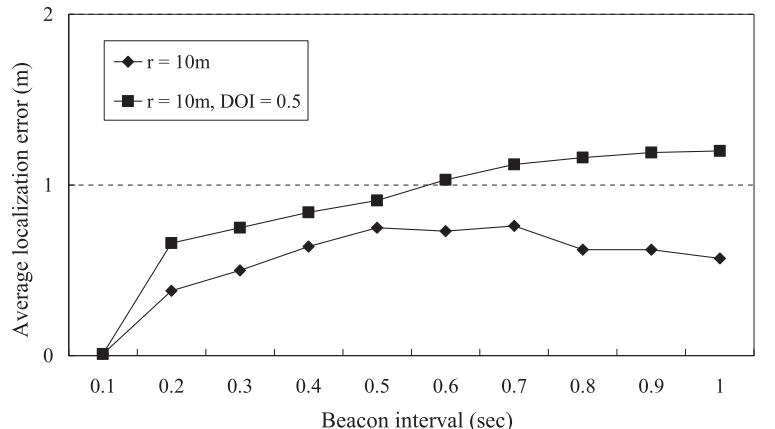

(a)

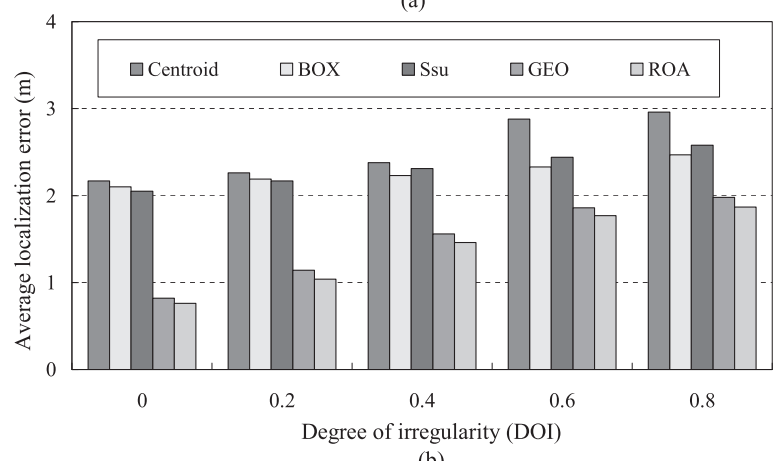

(b)

Fig. 11 Average localization error versus DOI model (a) ROA with $\mathrm{DOI}=0.5$ (b) different schemes with DOI $=0 \sim 0.8$.

DOI for different localization schemes with $r=20 \mathrm{~m}$, $\Delta \theta=30^{\circ}, \Delta t=0.2 \mathrm{sec}$, and $v=10 \mathrm{~m} / \mathrm{sec}$ is shown in Fig. 11. The average localization error using the DOI radio model is greater than for the normal model.

\subsection{Scheme Evaluation}

The schemes of Centroid [7] and BOX-constraint [9] are the popular conventional schemes in sensor localization. Ssu's scheme [18] is well-known and has been cited so many times recently and is served as a benchmark program in the field of sensor localization. We have added another localization scheme, GEO-constraint [12], an improved Ssu's scheme. So we evaluate the performance and compare ROA with these schemes.

- Average location errors: The results of the simulation show that the average location errors of ROA are below 1 in general and outperform those of the other three schemes. We take the results of the execution time in Fig. 12 (b). and compare these results in Fig. 12 (a).

- Average execution time: In the simulation, the average execution time for the different schemes depends on the values of average location error that we set. A comparison of average execution times between these localization schemes with the ROA scheme for $r=20 \mathrm{~m}$, $\Delta \theta=30^{\circ}$, and $v=10 \mathrm{~m} / \mathrm{sec}$ is shown in Fig. 12 (b) We set the terminating constraints on average localization errors depending on the properties of the scheme to avoid overly long execution times. For example, Centroid and Constraint are set for localization errors
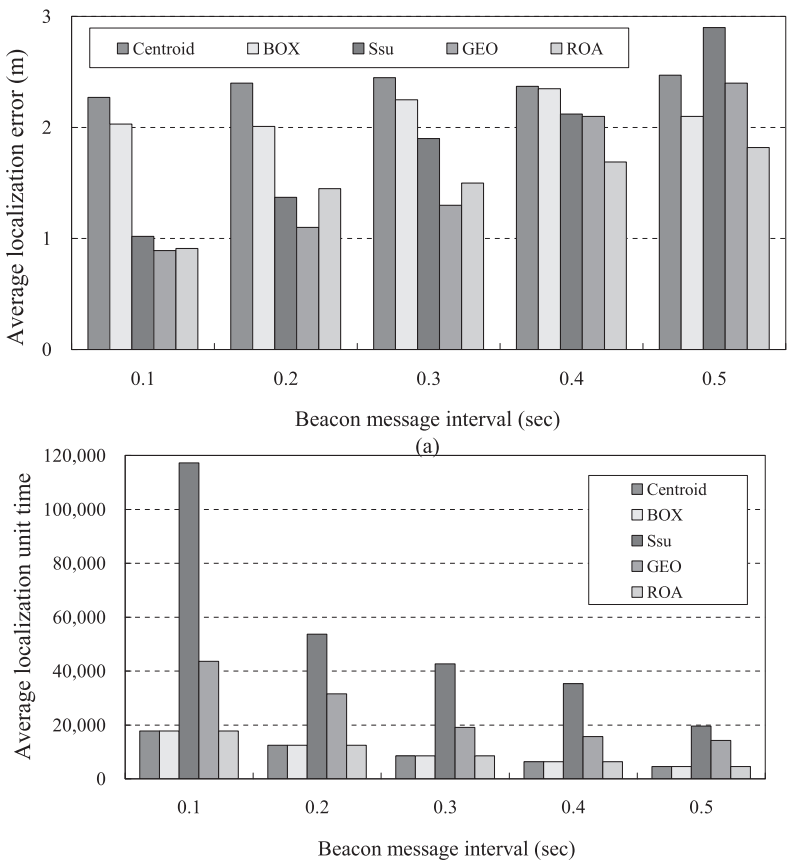

(b)

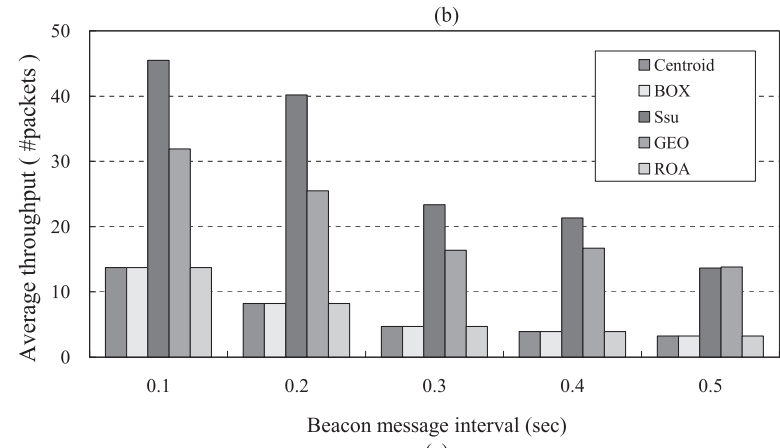

(c)

Fig. 12 Performance evaluation versus beacon interval for different schemes (a) localization error (b) localization time (c) throughput.

less than $3 \mathrm{~m}$, Ssu's scheme $2 \mathrm{~m}$, and our ROA scheme $1 \mathrm{~m}$. Based on these constraints, we find that the average execution time of Centroid and Constraint are similar. Ssu's execution time is similar to ROA, but Ssu's scheme location error is almost double that of ROA.

- Average throughput: Reducing the number of beacon message reduces the overheads for each sensor node. However, we still use the results of the execution time in Fig. 12 (b). and compare the results in Fig. 12 (c).

\section{Conclusion}

In this paper, we presented a simple and efficient rangefree localization scheme, called ROA, that can locate sensor nodes without requiring distance hardware and still achieve fine-grained accuracy. All computation is performed locally so the mechanism is distributed, scalable, effective, and power efficient. The proposed technique is supported by a mobile beacon with a directional antenna and GPS. The beacon moves along two traverse trajectories and periodically broadcasts its current information. Each sensor node 
receives the messages but does not interact with other nodes for localization. Distributed computation of node position involving elementary operations allows the system to operate in a low power consumption mode. Simulation results show that this mechanism outperformed previous range-free localization schemes.

\section{References}

[1] H.S. Abdelsalam and S. Olariu, "Passive localization using rotating anchor pairs in wireless sensor networks," Proc. 2nd ACM International Workshop on Foundations of Wireless Adhoc and Sensor Networking and Computing, FOWANC '09, pp.67-76, 2009.

[2] I.F. Akyildiz, W. Su, Y. Sankarasubramaniam, and E. Cayirci, "Wireless sensor networks: A survey," Comput. Netw., vol.38, no.4, pp.393-422, 2002.

[3] J.N. Al-Karaki and A.E. Kamal, "Routing techniques in wireless sensor networks: A survey," Wireless Comm., vol.11, no.6, pp.628, Dec. 2004.

[4] P. Bahl and V.N. Padmanabhan, "RADAR: An in-building RF-based user location and tracking system," Proc. IEEE Joint Conf. IEEE Computer Comm. Societies (INFOCOM), pp.775-784, 2000.

[5] A. Boukerche, H.A.B. F Oliveira, E.F. Nakamura, and A.A.F. Loureiro, "Localization systems for wireless sensor networks," IEEE Wireless Commun., vol.14, no.6, pp.6-12, 2007.

[6] J. Broch, D.A. Maltz, D.B. Johnson, Y.C. Hu, and J.G. Jetcheva, "A performance comparison of multi-hop wireless ad hoc network routing protocols," Proc. ACM Int. Conf. Mobile Computing Networking (MOBICOM), pp.85-97, 1998.

[7] N. Bulusu, J. Heidemann, and D. Estrin, "GPS-less low cost outdoor localization for very small devices," IEEE Pers. Commun. Mag., vol.7, no.5, pp.28-34, 2000.

[8] L. Doherty, K.S.J. Pister, and L.E. Ghaoui, "Convex position estimation in wireless sensor networks," Proc. IEEE INFOCOM '01, vol.3, pp.1655-1663, 2001.

[9] A. Galstyan, B. Krishnamachari, K. Lerman, and S. Pattem, "Distributed online localization in sensor networks using a moving target," Proc. Third Intel Symp. Information Processing in Sensor Networks (IPSN '04), pp.61-70, 2004.

[10] S. Guha, R. Murty, and E. Sirer, "Sextant: A unified node and event localization framework using non-convex constraints," ACM International Symposium on Mobile Ad Hoc Networking and Computing (IPSN '04), pp.205-216, 2005.

[11] T. He, C. Huang, B.M. Blum, J.A. Stankovic, and T. Abdelzher, "Range-free localization schemes for large scale sensor networks," Proc. ACM MobiCom '03, pp.81-95, Sept. 2003.

[12] S. Lee, E. Kim, C. Kim, and K. Kim, "Localization with a mobile beacon based on geometric constraints in wireless sensor networks," IEEE Trans. Wireless Commun., vol.8, no.12, pp.5801-5805, 2009.

[13] G. Mao, B. Fidan, and B.D.O. Anderson, "Wireless sensor network localization techniques," Comput. Netw., vol.51, no.10, pp.25292553, 2007.

[14] D. Niculescu and B. Nath, "Ad hoc positioning system (APS) using AOA," Proc. Global Telecomm. Conf. (Globecom '01), vol.1, pp.2926-2931, 2001.

[15] D. Niculescu and B. Nath, "DV based positioning in ad hoc networks," J. Tele. Systems, vol.22, pp.267-280, 2003.

[16] G.J. Pottie and W.J. Kaiser, "Wireless integrated network sensors," Commun. ACM, vol.43, no.5, pp.51-58, 2000.

[17] N.B. Priyantha, A. Chakraborty, and H. Balakrishnan, "The cricket location-support system," Proc. ACM MobiCom '00, pp.32-43, 2000.

[18] K.-F. Ssu, C.-H. Ou, and H.C. Jiau, "Localization with mobile anchor points in wireless sensor networks," IEEE Trans. Veh. Technol., vol.54, no.3, pp.1186-1197, 2005.

[19] Y.-H. Wu and W.-M. Chen, "Localization of wireless sensor net- works using a moving beacon with a directional antenna," Proc. 11th IEEE International Conference on HPCC, pp.237-242, 2009.

[20] B. Xiao, H. Chen, and S. Zhou, "Distributed localization using a moving beacon in wireless sensor networks," IEEE Trans. Parallel Distrib. Syst., vol.19, no.5, pp.587-600, May 2008.

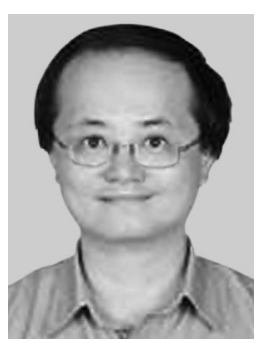

Yao-Hung Wu received the B.S. degree in computer science and information engineering from Tunghai University, Taiwan, and the M.S. degree in electronic engineering from Chung Yuan University, Taiwan. He is currently pursuing the Ph.D. degree in the Department of Electronic Engineering, National Taiwan University of Science and Technology, Taiwan. He had worked as a research assistant at Chung-Shan institute of Science and Technology, Ministry of National Defense. His research interests include wireless sensor networks and communication networks.

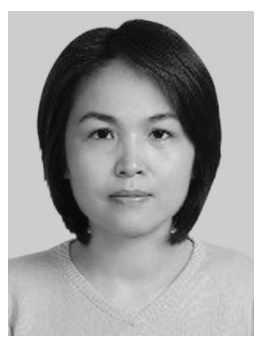

Wei-Mei Chen received her Ph.D. degree in computer science and information engineering from National Taiwan University in 2000. She is currently an associate professor at National Taiwan University of Science and Technology. Her research interests include algorithm design and analysis, automatic memory management, and mobile computing. 